\title{
Conservative integrated treatment of adhesive capsulitis of the shoulder
}

\author{
ADRIANO RUSSO', ANNALISA ARRIGHI², LUIGI VIGNALE³, LUIGI MOLFETTA² \\ IFCA Florence Institute of Care and Support, Florence, Italy \\ 2 Orthopaedics-Rehabilitation Unit, Department of Neuroscience, Rehabilitation, Ophthalmology, Genetics and \\ Maternal-Infantile Sciences (DINOGMI), University of Genoa, Italy \\ ${ }^{3}$ Alma Mater Clinic, La Spezia, Italy
}

\begin{abstract}
Purpose: the aim of this study was to present the results of a conservative treatment for adhesive capsulitis based on an original protocol of combined pharmacological and rehabilitation treatment.

Methods: fifty-two patients with idiopathic adhesive capsulitis were enrolled in the present study.

The treatment protocol included the use of hyaluronic acid and anaesthetic periarticular and intra-articular injections followed by a specific program of capsule and muscle stretching.

Results: the results of this treatment showed complete recovery of range of motion (ROM) in 50 of the 52 cases. The mean pre-treatment ROM values were: $85^{\circ}$ for forward elevation, $75^{\circ}$ for abduction, $25^{\circ}$ for external rotation, and $15^{\circ}$ for internal rotation. The posttreatment mean ROM values showed marked improvements: $175^{\circ}$ for forward elevation, $175^{\circ}$ for abduction, $87.5^{\circ}$ for external rotation and $75^{\circ}$ for internal rotation.
\end{abstract}

Conclusions: conservative treatment of adhesive capsulitis based on a combined pharmacological and rehabilitation approach was found to be effective in resolving pain and stiffness in $96 \%$ of the patients.

Level of evidence: level IV, therapeutic case series.

Key Words: adhesive capsulitis, drug therapy, hyaluronic acid, rehabilitation, shoulder.

\section{Corresponding Author:}

Adriano Russo, MD

IFCA Fiorentino Institute of Care and Support, Florence

Via Fieschi 400, 19131 La Spezia, Italy

Phone: +393482280980

E-mail: studiomedico.russo@libero.it

\section{Introduction}

Adhesive capsulitis is a condition affecting the glenohumeral joint characterized by pain and loss of active and passive joint mobility, due to the fibrosis of the joint capsule with the absence of adhesions, leading to decreased joint volume. It is generally primary or idiopathic and arises spontaneously in the absence of specific causes such as traumas, fractures, tendon injuries or dislocations, which are causes of secondary shoulder stiffness. In diabetic patients, the incidence of adhesive capsulitis ranges from 10 to $20 \%$ (10). The classic description of the disease is that of Codman (1) who, in 1934, defined "frozen shoulder" as a morbid condition characterized by muscle spasms and glenohumeral joint stiffness; ten years later, Neviaser (2) proposed the term "adhesive capsulitis", which certainly better reflects the pathophysiology of the disease, describing three clinical stages: the freezing stage, characterized by acute and continuous pain and arm stiffness in adduction and internal rotation; the frozen stage, during which there is less pain but more stiffness; and the thawing stage, during which wellbeing and movement are slowly and gradually restored. Even though this disease has a benign course, its symptoms can sometimes persist for a long time, possibly leading to functional damage of the shoulder girdle and general disability. The treatment of first choice for adhesive capsulitis is based on a rehabilitation therapy program that has to follow precise steps leading to functional recovery of the shoulder joint, avoiding the need for surgery (3). Conservative treatment combines rehabilitation with drug treatments, both general and local, e.g. intra-articular steroids and capsular distension induced by hyaluronic acid or associated treatments. The aim of this study was to present the results 
of a conservative treatment for adhesive capsulitis based on an original protocol that combines pharmacological and rehabilitation treatments.

\section{Methods}

Fifty-two patients with idiopathic adhesive capsulitis (IAC) were enrolled in the present study: 40 women (77\%) and 12 men (23\%), aged between 36 and 52 years. The right shoulder was affected in 18 cases (34.6\%) and the left shoulder in 34 cases (65.4\%); in 35 cases $(67.3 \%)$ the IAC involved the non-dominant side. The interval of time elapsing between the onset of the symptoms and the start of the treatment ranged from 3 to 12 months.

In 20 patients $(38.5 \%)$ the onset of the disease reportedly occurred following a mild trauma, which however was not considered to have been influential in the genesis of the disease; in the remaining 32 cases $(61.5 \%)$ onset occurred in the absence of trauma; 16 patients $(30.8 \%)$ had an endocrine or dysmetabolic disorder (hypo/hyperthyroidism, diabetes, hypercholesterolemia), sometimes mild, while four $(7.7 \%)$ had Sjögren syndrome, two $(3.8 \%)$ were under treatment with anticonvulsant drugs, four $(8 \%)$ were being treated for headache, four $(7.7 \%)$ were under treatment with anxiolytics or antidepressants, and 24 (46.1\%) had psychological profile characterized by anxiety. All the patients underwent standard plain radiographs of both shoulders and a magnetic resonance (MR) exam to assess lesions typical of IAC: thickening of the coracohumeral ligament, thickening of the joint capsule, especially in the rotator cuff interval, and obliteration of the space under the coracoid process (4). The integrated conservative therapy used in this study combined analgesic treatment and physiotherapy.

The drug therapy consisted of locoregional injections of $10 \mathrm{ml}$ of local anesthetic (ropivacaine $2 \mathrm{mg} / \mathrm{ml}$ ), administered in preparation for motor re-education, and intra-articular and subacromial injections of $2 \mathrm{ml}$ of low molecular-weight hyaluronic acid (Hyalgan; Fidia Farmaceutici, Abano Terme, Italy) via posterior, anterosuperior and lateral portals three times a week (Fig. 1). Seven cases (13.5\%) with severe joint restriction also underwent (in addition to the above-descri-
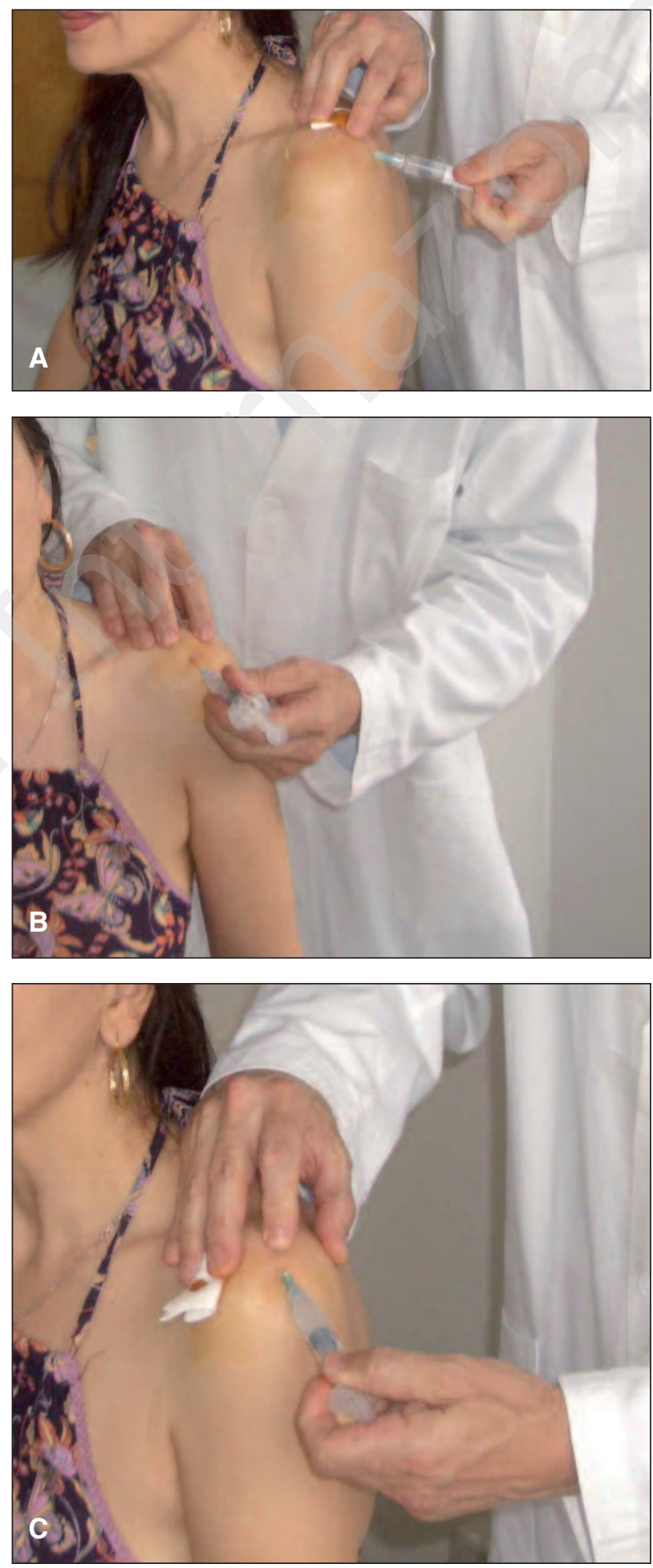

Fig. 1. A: Posterior access for subacromial and/or intra-articular injection. B: Anterosuperior access for subacromial injection. C: Lateral access for subacromial injection. 
bed therapy) anesthetic block of the suprascapular nerve and/or low doses of triamcinolone acetonide added to injections on account of its favorable effect on the sliding planes.

At the same time as receiving the analgesic injections, the patients also began the physiotherapy program consisting of capsule and muscle stretching exercises in all directions and unloaded exercises to promote recovery of passive movement (Fig. 2).

As regards the timing of the interventions, the protocol included three injections per week, each followed by a session of motor re-education (three per week). The patient was also assigned a series of self-assisted exercises to perform at home.
All the patients included in the study were examined before treatment and at follow-up. Passive range of motion (ROM) was evaluated before the treatment and again two weeks and six weeks following the final injection.

\section{Results}

The mean pre-treatment $\mathrm{ROM}$ values were: $85^{\circ}$ for forward elevation, $75^{\circ}$ for abduction, $25^{\circ}$ for external rotation, and $15^{\circ}$ for internal rotation. The post-treatment mean $\mathrm{ROM}$ values showed marked improvements: $175^{\circ}$ for forward elevation, $175^{\circ}$ for abduction,
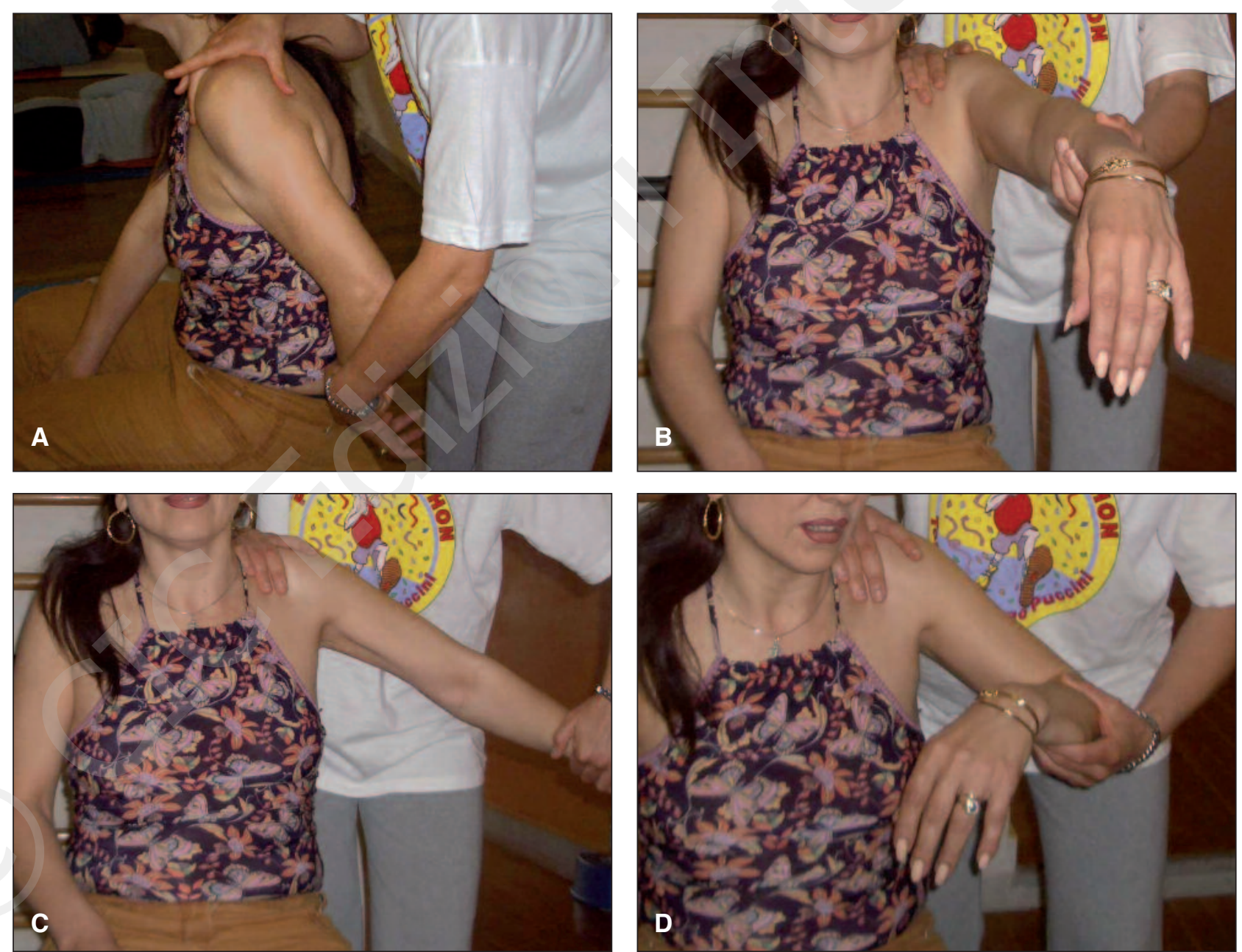

Fig. 2. Capsule and muscle stretching exercises in four directions and unloaded exercises for recovery of passive movement. A: Capsule stretching in internal rotation. B: Forward elevation with the scapula stabilized. C: Inferior capsule stretching in abduction. D: Stretching in external rotation with the scapula stabilized. 
$87.5^{\circ}$ for external rotation and $75^{\circ}$ for internal rotation.

A reduction of pain and a slow and gradual recovery of movement was observed in all the patients as from the first treatment sessions. Almost complete resolution of the symptoms and satisfactory recovery of ROM were obtained after a mean of 5-7 weeks and a mean of 1520 sessions of combined treatment.

Overall, 50 of the 52 patients (96.1\%) recovered, obtaining a good clinical outcome and reporting complete subjective satisfaction. No patient presented relapse of symptoms at the final follow-up. In two patients (3.8\%), affected by juvenile insulin-dependent diabetes, the disease was found to be resistant to treatment and these patients required arthroscopic capsular release.

\section{Discussion}

Adhesions between the capsule and neck of the humerus are the cause of IAC, which results in pain and severe joint stiffness. The anatomical and clinical similarities between adhesive capsulitis and Dupuytren's disease have prompted in-depth investigation of the pathogenesis of these two diseases, in order to establish the most appropriate therapeutic course. Bunker et al. (5) showed that fibroblastic proliferation and myoblastic transformation phenomena underlie capsulitis, similarly to what occurs in Dupuytren's disease. This correlation was subsequently confirmed by Uthoff and Boileau (6), who identified the presence, in capsular structures of the capsulitic shoulder, of vimentin, a cytocontractile protein present in pathological tissue in Dupuytren's disease.

The fibroblastic proliferative process involves the entire joint capsule, and the fact that anterior capsular release generally leads to resolution of the clinical picture has led to the suggestion that fibroplasia and contracture are two clearly distinct processes, and that joints stiffness might not be entirely related to capsular fibroplasia (7).

The effectiveness of conservative treatment of IAC is well documented in the literature. Griggs et al. (8) recorded a satisfactory outcome on objective and subjective clinical assessment at 22-month follow-up in
$90 \%$ of a series of 71 patients affected by IAC. Local analgesic treatment using anesthetic suprascapular nerve block and trigger point infiltrations has been employed as a method of diagnostic confirmation, and above all as a therapeutic tool in the treatment of idiopathic capsulitis (9). However, diabetic patients show a certain resistance to conservative treatment of adhesive capsulitis; morphological examination of pathological capsular tissue samples taken intra-operatively has shown the presence of newly vascularized synovial tissue, while immunohistochemical examination has revealed the presence of vascular endothelial growth factor, which has the capacity to induce intense synovial neovascularization (7). Furthermore, a high presence of intercellular adhesion molecule- 1 has been found in shoulder capsule tissue of diabetic patients, correlating with inflammation and fibrosis (10).

The presence of idiopathic adhesive capsulitis of the shoulder must, first of all, be established through a correct differential diagnosis versus secondary shoulder stiffness, which might not benefit from this type of treatment; patients with insulin-dependent diabetes and those who use anticonvulsant drugs are, in general, more resistant to conservative therapy.

The protocol used in the present study is supported by literature data on the use of combined rehabilitation and pharmacological approaches. Capsular distension induced by hyaluronic acid has been shown to be more effective than treatment with steroids, especially in terms of recovery of external rotation, assessed at two weeks and at six weeks after the last injection (11). Harris e al. (12) pointed out that the use of intra-articular hyaluronic acid is safer than the use of intra-articular corticosteroid injections in the treatment of IAC. Hsieh et al. (13) found that intra-articular injection of hyaluronic acid did not seem to offer additional benefits over the use of physical therapy, although it reduced the need for further drug treatment. Conversely, Dehghan et al. (14) did not find statistically significant differences between oral drug therapy and intra-articular administration of corticosteroids in diabetic patients, while physical therapy (transcutaneous electrical nerve stimulation, thermotherapy, manual therapy) seemed to show greater efficacy if associated with bee venom acupuncture (15). Thomas et al. (16) reported good 
results following treatment of IAC with manipulation under anaesthetic (MUA) and injection of corticosteroid and local anesthetic, irrespective of the disease duration.

All the various treatments for IAC (steroid or hyaluronic acid injections, physical therapy, acupuncture, MUA and arthroscopic distension and capsular release) have also been considered from a cost-effectiveness perspective in relation to resolution of pain and dysfunction. This analysis, albeit producing results described as "very uncertain", seemed to show that steroid injections may be more cost-effective than steroid injections combined with physiotherapy or physiotherapy alone (17). This finding has been confirmed in diabetic patients in particular (18).

In our study we combined the two approaches - rehabilitation and injections - opting to use low molecular-weight hyularonic acid associated with local anesthetic because of the favorable effect it is known to have on connective tissue elasticity. In the most severe cases, the addition of small doses of triamcinolone acetonide exploits the favorable effect on the sliding planes. The results of our study - resolution of the clinical picture in $96.1 \%$ of cases after a mean of 5-7 weeks of treatment - were found to be superior to ones previously reported in the literature $(4,11,13,15,17$, 19). Therefore, in our opinion, surgical treatment of adhesive capsulitis should be reserved for cases resistant to conservative treatment, which in the present study accounted for only $3.8 \%$ of the treated patients.

\section{References}

1. Codman EA. The shoulder: rupture of the supraspinatus tendon and other lesions in or about the subacromial bursa. Thomas Todd Co., Boston, 1934.

2. Neviaser JS. Adhesive capsulitis of the shoulder: a study of the pathological findings in periarthritis of the shoulder. J Bone Joint Surg Am. 1945;27:211-222.

3. Donatelli R. Physical therapy of the shoulder, 5th ed. Elsevier-Churchill Livingston Elsevier, St Louis, 2012.

4. Zhao W, Zheng X, Liu Y, Yang W, Amirbekian V, Diaz LE, Huang X. An MRI study of symptomatic adhesive capsulitis. PLoS One. 2012;7:e47277.

5. Bunker TD, Anthony PP. The pathology of frozen shoulder.
A Dupuytren-like disease. J Bone Joint Surg Br. 1995;77:677683.

6. Uthoff HK, Boileau P. Primary frozen shoulder: global capsular stiffness versus localized contracture. Clin Orthop Relat Res. 2007;456:79-84.

7. Ryu JD, Kirpalani PA, Kim JM, Nam KH, Han CW, Han $\mathrm{SH}$. Expression of vascular endothelial growth factor and angiogenesis in the diabetic frozen shoulder. J Shoulder Elbow Surg. 2006;15: 679-685.

8. Griggs SM, Ahn A, Green A. Idiopathic adhesive capsulitis. A prospective functional outcome study of nonoperative treatment. J Bone Joint Surg Am. 2000;82-A:1398-1407.

9. Jankovic D, van Zundert A. The frozen shoulder syndrome. Description of a new technique and five case reports using the subscapular nerve block and subscapularis trigger point infiltration. Acta Anestesiol Belg. 2006;57:137-143.

10. Kim YS, Kim JM, Lee YG, Hong OK, Kwon HS, Ji JH. Intercellular adhesion molecule-1 (ICAM-1, CD 54) is increased in adhesive capsulitis. Joint Surg Am. 2013;95:e181-188.

11. Park KD, Nam HS, Lee JK, Kim YJ, Park Y. Treatment effects of ultrasound-guided capsular distension with hyaluronic acid in adhesive capsulitis of the shoulder. Arch Phys Med Rehabil. 2013;94:264-270.

12. Harris JD, Griesser MJ, Copelan A, Jones GL. Treatment of adhesive capsulitis with intra-articular hyaluronate: a systematic review. Int J Shoulder Surg. 2011;5:31-37.

13. Hsieh LF, Hsu WC, Lin YJ, Chang HL, Chen CC, Huang V. Addition of intra-articular hyaluronate injection to physical therapy program produces no extra benefits in patients with adhesive capsulitis of the shoulder: a randomized controlled trial. Arch Phys Med Rehabil. 2012;93:957-964.

14. Dehghan A, Pishgooei N, Salami MA, Zarch SM, NafisiMoghadam R, Rahimpour S, Soleimani H, Owlia MB. Comparison between NSAID and intra-articular corticosteroid injection in frozen shoulder of diabetic patients; a randomized clinical trial. Exp Clin Endocrinol Diabetes. 2013;121:75-79.

15. Koh PS, Seo BK, Cho NS, Park HS, Park DS, Baek YH. Clinical effectiveness of bee venom acupuncture and physiotherapy in the treatment of adhesive capsulitis: a randomized controlled trial. J Shoulder Elbow Surg. 2013;22:1053-1062.

16. Thomas WJ, Jenkins EF, Owen JM, Sangster MJ, Kirubanandan R, Beynon C, Woods DA. Treatment of frozen shoulder by manipulation under anaesthetic and injection: does the timing of treatment affect the outcome? Bone Joint Surg Br. 2011;93:1377-1381.

17. Maund E, Craig D, Suekarran S, Neilson A, Wright K, Brealey S, Dennis L, Goodchild L, Hanchard N, Rangan A, Richardson G, Robertson J, McDaid C. Management of frozen shoulder: a systematic review and cost-effectiveness analysis. Health Technol Assess. 2012;16:1-264.

18. Roh YH, Yi SR, Noh JH, Lee SY, Oh JH, Gong HS, Baek $\mathrm{GH}$. Intra-articular corticosteroid injection in diabetic patients with adhesive capsulitis: a randomized controlled trial. Knee Surg Sports Traumatol Arthrosc. 2012;20:19471952.

19. Wang K, Ho V, Hunter-Smith DJ, Beh PS, Smith KM, Weber AB. Risk factors in idiopathic adhesive capsulitis: a case control study. J Shoulder Elbow Surg. 2013;22:e24-29. 